\title{
An Alternative Approach for Nonlinear Optimization Problem with Caputo - Fabrizio Derivative
}

\author{
Firat Evirgen ${ }^{1}$, and Mehmet Yavuz ${ }^{2, *}$ \\ ${ }^{1}$ Department of Mathematics, Balikesir University, Balikesir, Turkey \\ ${ }^{2}$ Department of Mathematics-Computer Sciences, Necmettin Erbakan University, Konya, Turkey
}

\begin{abstract}
In this study, a fractional mathematical model with steepest descent direction is proposed to find optimal solutions for a class of nonlinear programming problem. In this sense, Caputo-Fabrizio derivative is adapted to the mathematical model. To demonstrate the solution trajectory of the mathematical model, we use the multistage variational iteration method (MVIM). Numerical simulations and comparisons on some test problems show that the mathematical model generated using Caputo-Fabrizio fractional derivative is both feasible and efficient to find optimal solutions for a certain class of equality constrained optimization problems.
\end{abstract}

\section{Introduction}

Optimization theory and fractional calculus are the essential elements of the mathematical modeling process. Because of this reason, they are the most attractive two research areas studied by scientists in the word.

The optimization concept is applied for representing real-world problems, which aims to minimize and maximize some parameters, using mathematical models. Transportation systems, production processes, creating portfolios, routing of traffic, a system controlling and etc. al. all are examples of the optimization concept in our life. In particular, incredibly many new methods have been introduced in the literature for solving these type of problems, within the past twenty years. Gradient-based dynamic system method is one of these methods and was first introduced by Arrow and Hurwicz in 1958 [1]. In this method, the solution process is generated with the help of a differential equation system which includes optimality conditions. In recent past, Wang et al. [2], Jin et al. [3], Shikhman and Stein [4] and, Özdemir and Evirgen [5] have considered a gradient-based system for the different type of optimization problems.

\footnotetext{
*Corresponding author: mehmetyavuz@konya.edu.tr
} 
On the other hand, the fractional calculus topics are drawn increasing attention from the researchers as it has a capability of representing real-world problems better than regular derivative. In this sense, various types of fractional derivatives, integrals, and applications are introduced by scientists. The Riemann-Liouville's fractional derivative and the Caputo fractional derivative are the most popular definitions in the literature. Podlubny [6] and Baleanu et al. [7] provided comprehensive discussions on these definitions. In their recent work, Caputo and Fabrizio [8] introduced a new derivative of fractional order with a nonsingular kernel. Some interesting features and implementations of the new fractional derivative operator are introduced by Caputo and Fabrizio [9] and Losada and Nieto [10].

In the last two years, many authors used Caputo-Fabrizio fractional derivative operator in many interesting models and with many known methods, see [11-22].

In the present work, we will investigate the solution procedure of the optimization problems with the constructed mathematical model by Caputo-Fabrizio fractional derivative operator. To demonstrate the solution, we use the multistage variational iteration method (MVIM).

In this sense, the remainder paper is organized as follows. We present in Section 2 some basic notion and theoretical aspects of the optimization theory, Caputo-Fabrizio fractional derivative and variational iteration method. The newly defined mathematical model with Caputo-Fabrizio fractional derivative and the solution procedure by MVIM are given in Section 3, followed by the presentation of the solutions obtained by the proposed mathematical model and MVIM for well-known benchmarks.

\section{Some Basic Definitions and Theorems}

\subsection{Fundamentals of optimization theory}

To explain basic notions of the optimization theory let consider the following optimization problem which contains equality constraints,

$$
\begin{array}{ll}
\min _{x \in \Re^{n}} & f(x), \\
\text { s.t. } & h(x)=0,
\end{array}
$$

where $f: \mathfrak{R}^{n} \rightarrow \mathfrak{R}, h: \mathfrak{R}^{n} \rightarrow \mathfrak{R}^{p}$ are functions. With the aid of the penalty function concepts, the equality constrained optimization problem is converted into the following optimization problem without constrained

$$
\min F(x, \eta)=f(x)+\underbrace{\eta \frac{1}{\gamma} \sum_{i=1}^{p}\left(h_{i}(x)\right)^{\gamma}}_{\text {Penalty function }} \text { s.t. } x \in \mathfrak{R}^{n} .
$$

where $\eta>0$ is a sufficiently large auxiliary penalty variable and $\gamma>0$ is a constant [23]. When $\gamma=2$ is taken, it is named as quadratic penalty function method. The following theorem shows us the convergence between the problem (1) and the problem (2) solutions. 
Theorem 1 Let $\left\{x^{(n)}\right\}$ be a solution sequence of the unconstrained optimization problems generated by the penalty function method (2) as $\eta \rightarrow \infty$. Then any accumulation points of the sequence are the solution of the original constrained problem (1).

\subsection{Caputo-Fabrizio fractional derivative}

Basic tools of the Caputo-Fabrizio fractional derivative of order $\alpha$ can be summarized as follow [8-10].

Definition 1 Let $f:[a, b] \rightarrow \mathfrak{R}$ be a continuous function. The left and the right Caputo fractional derivatives of order $\alpha>0$ are defined, respectively, by

$$
{ }^{C} D_{a^{+}}^{\alpha} f(t)=\frac{1}{\Gamma(m-\alpha)} \int_{a}^{t}(t-\tau)^{m-\alpha-1} f^{(m)}(\tau) d \tau, t \in(a, b]
$$

and

$$
{ }^{C} D_{b^{-}}^{\alpha} f(t)=\frac{1}{\Gamma(m-\alpha)} \int_{t}^{b}(-1)^{n}(t-\tau)^{m-\alpha-1} f^{(m)}(\tau) d \tau, t \in[a, b),
$$

where $\alpha$ is the order of derivative, and $n=[\alpha]+1$ with $[\alpha]$ being the integer part of $\alpha$

According to the above classical Caputo fractional derivative definition, novel fractional derivative definition consisting of the non-singular kernel can be given by the following definition.

Definition 2 Let $\alpha \in(0,1), f(x)$ be a function such that $f(x) \in H^{1}(a, b), a<b$ and $M(\alpha)$ be a normalization function such that $M(0)=M(1)=1$. The left Caputo-Fabrizio derivative of order $\alpha$ is defined as

$$
{ }^{C F} D_{a^{+}}^{\alpha} f(t)=\frac{M(\alpha)}{1-\alpha} \int_{a}^{t} f^{\prime}(\tau) \exp \left[-\frac{\alpha(t-s)}{1-\alpha}\right] d s .
$$

where $M(\alpha)$ is normalization function such that $M(0)=M(1)=1$ and, $0<\alpha \leq 1$.

Remark 1 The Caputo-Fabrizio fractional derivative is obtained by changing the kernel $(t-\tau)^{-\alpha}$ in Caputo fractional derivative formula with the function $\exp \left[-\frac{\alpha(t-\tau)}{1-\alpha}\right]$ and $\frac{1}{\Gamma(1-\alpha)}$ with $\frac{M(\alpha)}{1-\alpha}$ 
Remark 2 The Caputo-Fabrizio fractional derivative, is zero where $f$ as constant, as in the usual Caputo derivative. But, contrary to the usual Caputo fractional derivative, the kernel does not have singularity for $t=\tau$

In (2015), Losada and Nieto have redefined the Caputo-Fabrizio fractional derivative by taking $M(\alpha)=1$ as follow:

$$
{ }^{C F} D_{a^{+}}^{\alpha} f(t)=\frac{1}{1-\alpha} \int_{a}^{t} f^{\prime}(\tau) \exp \left[-\frac{\alpha(t-s)}{1-\alpha}\right] d s
$$

Furthermore, by using the Laplace transform for the general form of fractional differential equation involving Caputo-Fabrizio fractional derivative operator, they are stated the Caputo-Fabrizio fractional integral definition for $0<\alpha \leq 1$ as follow:

$$
{ }^{C F} I_{t}^{\alpha} f(t)=\frac{1-\alpha}{M(\alpha)} f(t)+\frac{\alpha}{M(\alpha)} \int_{a}^{t} f(\tau) d \tau
$$

Remark 3 When $0<\alpha \leq 1$, the property below convinces

$$
\left({ }^{C F} I_{t}^{\alpha}\right)\left({ }^{C F} D_{t}^{\alpha}\right) f(t)=f(t)-f(0)
$$

\subsection{Variational iteration method}

Let us consider the following general nonlinear differential equation to express the variational iteration method (VIM),

$$
L(u(t))+N(u(t))=g(t)
$$

where $L$ is a linear operator, $N$ is a nonlinear operator and $g(t)$ is a known analytical function [24].

We can construct a correction functional for (4) as follows,

$$
u_{i, k+1}(t)=u_{i, k}(t)+\int_{t_{0}}^{t} \lambda(\tau)\left\{L\left(u_{i, k}(\tau)\right)+N\left(\tilde{u}_{i, k}(\tau)\right)-g(\tau)\right\} d \tau
$$

where $\lambda$ is a general Lagrange multiplier, and $\tilde{u}_{i, k}$ is a restricted variation with $\delta \tilde{u}_{i, k}=0$. Thereby the exact solution can be obtained as

$$
u_{i}(t)=\lim _{k \rightarrow \infty} u_{i, k}(t)
$$




\section{A mathematical model involving Caputo-Fabrizio derivative}

Let consider the equality constrained optimization problem described by (1). As mentioned above, the optimization problem (1) is converted to the unconstrained optimization problem (2) by using the quadratic penalty function for $\gamma=2$.

In this work, a mathematical model, which is constructed by using the Caputo-Fabrizio fractional derivative, is handled for determining the optimal solutions of (2), and also of the equality constrained optimization problem (1) with the help of the MVIM. This kind of fractional mathematical model for solving optimization problems was first introduced by Evirgen and Özdemir [25-28].

The mathematical model involving the Caputo-Fabrizio derivative can be defined by the following expression:

$$
\begin{array}{ll}
{ }^{C F} D_{t}^{\alpha} x(t)=-\nabla_{x} F(x, \eta) & , 0<\alpha \leqslant 1, \\
x_{i}(0)=x_{i} 0 & , i=1 \ldots n .
\end{array}
$$

where $\nabla_{x} F(x, \eta)$ is the gradient vector of (2) with respect to the $x \in \mathfrak{R}^{n}$.

Definition 4 A point $x^{*}$ is referred to as an equilibrium point of (5) if it satisfies the righthand side of Equation (5).

Without loss of generality, the mathematical model (5) can be rearranged by the following formula as follow

$$
{ }^{C F} D_{t}^{\alpha} x_{i}(t)=g_{i}\left(t, \eta, x_{1}, x_{2}, \ldots, x_{n}\right), i=1,2, \ldots, n
$$

In this stage, the MVIM algorithm is applied to acquire the stable equilibrium point of the fractional model (6). The MVIM can be described in terms of certain modifications to VIM. In this approach, the integral interval $\left[t_{0}, t\right)$ is divided into the equal subinterval such as $\left[t_{0}, t_{1}\right),\left[t_{1}, t_{2}\right), \ldots,\left[t_{j-1}, t_{j}=t\right)$ to ensure the validity of the approximations of VIM in the large interval. The multistage technique is first adapted to the VIM by Batiha and et al. [29].

The correction functional for (6) according to the MVIM can be constructed as

$$
x_{i}^{k+1}(t)=x_{i}^{k}(t)+I_{t}\left(\lambda_{i}(\tau)\left({ }^{C F} D_{t \alpha}^{\alpha} x_{i}^{k}(\tau)-g_{i}\left(\tilde{x}_{1}^{k}(\tau), \ldots, \tilde{x}_{n}^{k}(\tau)\right)\right)\right) d \tau
$$

where $\lambda_{i}(i=1,2, \ldots, n)$ are general Lagrange multiplier and $\tilde{x}_{1}, \tilde{x}_{2}, \ldots, \tilde{x}_{n}$ denote restricted variations such that $\delta \tilde{x}_{i}=0$. 
To identify the multiplier, we take the usual Riemann integral instead of Caputo-Fabrizio fractional integral and taking variation with respect to the independent variable $x_{i}$, $i=1,2, \ldots, n$ with $\delta x_{i}\left(t^{*}\right)=0$,

$$
\delta x_{i}^{k+1}(t)=\delta x_{i}^{k}(t)+\delta \int_{t^{*}}^{t} \lambda_{i}(\tau)\left(x_{i}^{\prime k}(\tau)-g i\left(\tilde{x}_{1}^{k}(\tau), \ldots, \tilde{x}_{n}^{k}(\tau)\right)\right) d \tau
$$

and consequently we get the following stationary conditions:

$$
\begin{aligned}
& \left.\lambda_{i}^{\prime}(\tau)\right|_{\tau=t}=0 \\
& 1+\left.\lambda_{i}(\tau)\right|_{\tau=t}=0
\end{aligned}
$$

for $i=1,2, \ldots, n$. Thus the Lagrange multipliers can be determined by the following equality

$$
\lambda_{i}=-1, i=1,2, \ldots, n
$$

In this way, we acquire the MVIM formula by substituting the Lagrange multipliers (8) into the correctional functional (7) as follow:

$$
x_{i}^{k+1}(t)=x_{i}^{k}(t)-I_{t}\left({ }^{C F} D_{t}^{\alpha} x_{i}^{k}(\tau)-g_{i}\left(\tilde{x}_{1}^{k}(\tau), \ldots, \tilde{x}_{n}^{k}(\tau)\right)\right) d \tau, \quad i=1,2, \ldots, n
$$

If we begin with initial conditions $x_{i}\left(t^{*}\right)=x_{i}\left(t_{0}\right)=x_{i}(0)$, the iteration formula of the MVIM (9) can be carried out within every subinterval of equal length $\Delta t$, and so all solutions $x_{i}^{k}(t),(i=1,2, \ldots, n ; k=1,2, \ldots)$ are completely determined.

\section{Numerical simulations}

In this section, we give two examples to illustrate the effectiveness and applicability of the proposed fractional mathematical model involving the Caputo-Fabrizio derivative (6). The MVIM is applied and compared with the VIM and fourth order Runge-Kutta method (RK4).

Example 1 Consider the following nonlinear programming problem [30],

$$
\begin{array}{ll}
\text { minimize } & f(x)=100\left(x_{1}^{2}-x_{2}\right)^{2}+\left(x_{1}-1\right)^{2} \\
\text { subject to } & h(x)=x_{1}\left(x_{1}-4\right)-2 x_{2}+12=0
\end{array}
$$

Firstly, the problem (10) is transformed to the unconstrained optimization problem (2) with the quadratic penalty function and so we obtain

$$
F(x, \eta)=100\left(x_{1}^{2}-x_{2}\right)^{2}+\left(x_{1}-1\right)^{2}+\frac{1}{2} \eta\left(x_{1}\left(x_{1}-4\right)-2 x_{2}+12\right)^{2}
$$


where $\eta \in \mathfrak{R}^{+}(\eta \rightarrow \infty)$ is an auxiliary penalty variable. Thereafter, the mathematical model from (5) is defined as

$$
\begin{aligned}
&{ }^{C F} D_{t}^{\alpha} x_{1}(t)=-400\left(x_{1}^{2}-x_{2}\right) x_{1}-2\left(x_{1}-1\right) \\
&-\eta\left(2 x_{1}-4\right)\left(x_{1}^{2}-4 x_{1}-2 x_{2}+12\right), \\
&{ }^{C F} D_{t}^{\alpha} x_{2}(t)=200\left(x_{1}^{2}-x_{2}\right)+2 \eta\left(x_{1}^{2}-4 x_{1}-2 x_{2}+12\right), \\
& x_{1}(0)=0, x_{2}(0)=0
\end{aligned}
$$

where $0<\alpha \leqslant 1$.

Finally, in order to obtain the solutions of (11), the MVIM and VIM are implemented with auxiliary penalty variable $\eta=800$ and step size $\Delta T=0.1 \times 10^{-4}$ by using the MVIM recursive formula (9).
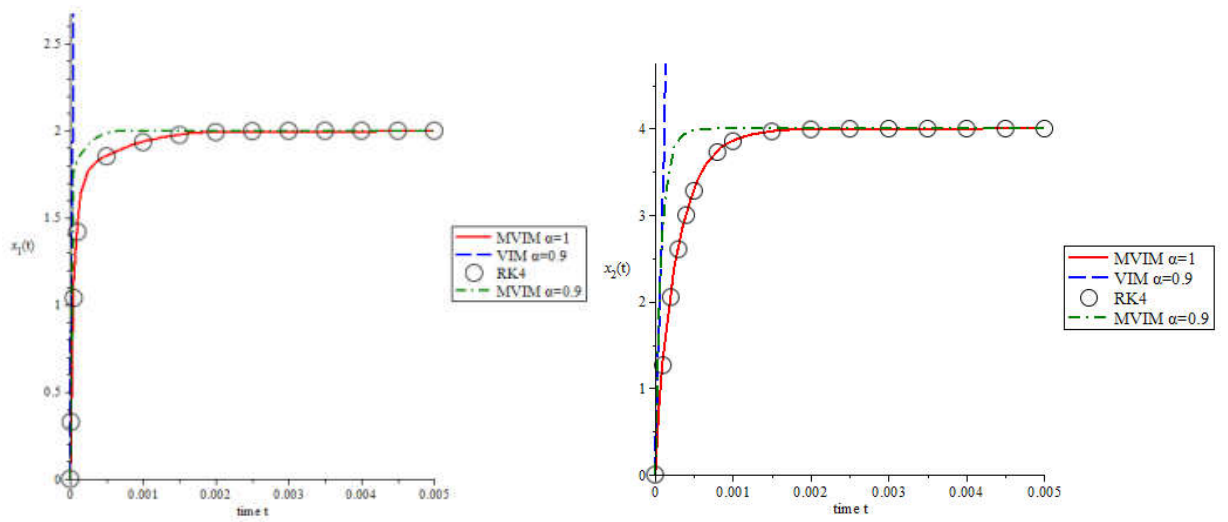

Fig.1. Numerical evaluation of $x(t)$ for problem (10) with MVIM, VIM and RK4.

In Figure 1, it can clearly be seen that the fractional model with the Caputo-Fabrizio fractional operator reaches the expected optimal solutions of (10) only one iteration using the MVIM. It can also be verified that MVIM with the fractional order has obtained the optimal solutions earlier than the VIM and RK4 with the integer order version.

Example 2 Let us consider the equality constrained problem in [30] as follow,

$$
\begin{array}{ll}
\text { minimize } & f(x)=\left(x_{1}-20\right)^{2}+\left(x_{2}+20\right)^{2}, \\
\text { subject to } & h(x)=\frac{x_{1}^{2}}{100}+\frac{x_{2}^{2}}{4}-1=0 .
\end{array}
$$

This is a practical problem and the best known optimal solution is $x_{1}^{*}=9.395$ and $x_{2}^{*}=-0.6846$. Thereafter, the corresponding mathematical model from (5) is defined as 


$$
\left.\begin{array}{l}
{ }^{C F} D_{t}^{\alpha} x_{1}(t)=2 x_{1}-40+\eta\left(\frac{1}{5000} x_{1}^{3}+\frac{1}{200} x_{1} x_{2}^{2}-\frac{1}{50} x_{1}\right), \\
{ }^{C F} D_{t}^{\alpha} x_{2}(t)=2 x_{2}+40+\eta\left(\frac{1}{200} x_{2} x_{1}^{2}+\frac{1}{8} x_{2}^{3}-\frac{1}{2} x_{2}\right) \\
x_{1}(0)=0, x_{2}(0)=0,
\end{array}\right\}
$$

where $0<\alpha \leqslant 1$ is the order of the Caputo-Fabrizio fractional derivative. The optimal solution of the problem (12) is obtained by utilizing the MVIM with the auxiliary penalty variable $\eta=6 \times 10^{5}$ and step size $\Delta T=0.1 \times 10^{-5}$. Hence, the solutions can be summarized in Tables 1 .

Table 1. The numerical evaluation of $x(t)$ for problem (12) with MVIM, VIM and RK4.

\begin{tabular}{ccccccc}
\hline \hline & \multicolumn{2}{c}{ MVIM $(\alpha=0.9)$} & \multicolumn{2}{c}{ MVIM $(\alpha=1)$} & \multicolumn{2}{c}{ RK4 $(\alpha=1)$} \\
\hline$t$ & $x_{1}(t)$ & $x_{2}(t)$ & $x_{1}(t)$ & $x_{2}(t)$ & $x_{1}(t)$ & $x_{2}(t)$ \\
\hline 0 & 0 & 0 & 0 & 0 & 0 & 0 \\
0.1 & 9.3330 & -0.7186 & 3.4920 & -1.8741 & 3.4919 & -1.8741 \\
0.2 & 9.3966 & -0.6847 & 6.0764 & -1.5885 & 6.0764 & -1.5885 \\
0.3 & 9.3967 & -0.6846 & 7.8531 & -1.2383 & 7.8530 & -1.2383 \\
0.4 & 9.3967 & -0.6846 & 8.8690 & -0.9242 & 8.8690 & -0.9242 \\
0.5 & 9.3967 & -0.6846 & 9.2690 & -0.7510 & 9.2690 & -0.7510 \\
\hline \hline
\end{tabular}

In the numerical simulation, we see that the VIM solutions for $\alpha=0.9$ are divergent. Furthermore, as we saw in the previous example, although the fractional order MVIM approaches the optimal solution at $t=0.5$, other methods have not yet been approached the desired optimal solution.

\section{Conclusion}

In this paper, a fractional mathematical model with the newly defined Caputo-Fabrizio fractional derivative is designed successfully for solving a certain class of equality constrained optimization problem. For the purpose of obtaining the optimal solutions, the steepest descent direction is integrated into the mathematical model. In addition to this, the solution trajectory of the generated fractional mathematical model is shown by applying the MVIM. Numerical comparisons between the RK4 and MVIM, VIM with the fractional and integer order are shown that the Caputo-Fabrizio fractional gradient based mathematical model is more reliable and stable than the integer order one for obtaining the optimal solutions of this type optimization problem (1). 


\section{References}

1. K.J. Arrow, L. Hurwicz and H. Uzawa, Studies in Linear and Non-Linear Programming, Stanford University Press, California (1958).

2. S. Wang, X.Q. Yang and K.L. Teo, A unified gradient flow approach to constrained nonlinear optimization problems, Comput. Optim. Appl., 25, pp. 251-268, (2003).

3. L. Jin, L.-W. Zhang and X. Xiao, Two differential equation systems for equality-constrained optimization, Appl. Math. Comput., 190, pp. 1030-1039, (2007).

4. V. Shikhman and O. Stein, Constrained optimization: projected gradient flows, J. Optim. Theory Appl., 140, pp. 117-130, (2009).

5. N. Özdemir and F. Evirgen, A dynamic system approach to quadratic programming problems with penalty method, Bull. Malays. Math. Sci. Soc., 33, pp. 79-91, (2010).

6. I. Podlubny, Fractional Differential Equations, Academic Press, New York (1999).

7. D. Baleanu, K. Diethelm, E. Scalas and J.J. Trujillo, Fractional calculus: models and numerical methods, Series on complexity, nonlinearity and chaos, World Scientific, Singapore, (2012)

8. M. Caputo and M. Fabrizio, A new definition of fractional derivative without singular kernel, Progr. Fract. Differ. Appl., 1, pp. 73-85, (2015).

9. M. Caputo and M. Fabrizio, Applications of new time and spatial fractional derivatives with exponential kernels, Progr. Fract. Differ. Appl., 2, pp. 1-11, (2016).

10. J. Losada and J.J. Nieto, Properties of a new fractional derivative without singular kernel, Progr. Fract. Differ. Appl., 1, pp. 87-92, (2016).

11. A. Atangana, B.S.T. Alkahtani, New model of groundwater flowing within a confine aquifer: Application of Caputo-Fabrizio derivative, Arab. J. Geosci., 9, pp. 1-8, (2016)

12. J. Singh, D. Kumar, J.J. Nieto, Analysis of an El Nino-Southern Oscillation model with a new fractional derivative, Chaos Solitons Fractals, 99, pp. 109-115, (2017).

13. I. Koca, A. Atangana, Solutions of Cattaneo-Hristov model of elastic heat diffusion with Caputo-Fabrizio and Atangana-Baleanu fractional derivatives, Therm. Sci., 21, pp. 22992305, (2017).

14. J. Hristov, Steady-state heat conduction in a medium with spatial non-singular fading memory: Derivation of Caputo-Fabrizio space-fractional derivative with Jeffrey's kernel and analytical solutions, Therm. Sci., 21, pp. 827-839, (2017).

15. M.S. Aydogan, D. Baleanu, A. Mousalou, and et al., On high order fractional integrodifferential equations including the Caputo-Fabrizio derivative, Bound Value Probl., 2018: 90, pp. 1-15, (2018).

16. M. Yavuz, N. Özdemir, A different approach to the European option pricing model with new fractional operator. Mathematical Modelling of Natural Phenomena, 13(1), pp. 1-12, (2018).

17. S. Ullah, M. Altaf Khan and M. Farooq, A new fractional model for the dynamics of the hepatitis B virus using the Caputo-Fabrizio derivative, Eur. Phys. J. Plus, 133: 237, (2018).

18. N.A. Asif, Z. Hammouch, M.B. Riaz and et al., Analytical solution of a Maxwell fluid with slip effects in view of the Caputo-Fabrizio derivative, Eur. Phys. J. Plus, 133: 272, (2018).

19. M.A. Dokuyucu, E. Celik, H. Bulut and et al., Cancer treatment model with the CaputoFabrizio fractional derivative, Eur. Phys. J. Plus 133: 92, (2018).

20. M. Yavuz, N. Özdemir, European vanilla option pricing model of fractional order without singular kernel, Fractal and Fractional, 2(1), 3, (2018).

21. A. Yokus, Numerical solutions of time fractional Korteweg-de vries equation and its stability analysis, Commun. Fac. Sci. Univ. Ank. Ser. A1 Math. Stat., 68(1), pp. 353-361, (2019).

22. A. Yokus, D. Kaya, Numerical and exact solutions for time fractional Burgers' equation, J. Nonlinear Sci. Appl., 10(7), pp. 3419-3428, (2017).

23. D.G. Luenberger and Y. Ye, Linear and Nonlinear Programming, Third Edition, Springer, New York (2008).

24. J.H. He, Variational iteration method for delay differential equations, Commun. Nonlinear Sci. Numer. Simul., 2, pp. 235-236, (1997). 
25. F. Evirgen and N. Özdemir, Multistage Adomain decomposition method for solving NLP problems over a nonlinear fractional dynamical system. J. Comput. Nonlinear Dyn., 6, 021003 (2011).

26. F. Evirgen and N. Özdemir, A fractional order dynamical trajectory approach for optimization problem with HPM, Fractional Dynamics and Control, Springer, Eds. Baleanu, D., Machado, J.A.T., Luo, A.C.J., pp. 145-155 (2012).

27. F. Evirgen, Analyze the optimal solutions of optimization problems by means of fractional gradient based system using VIM, An International Journal of Optimization and Control: Theories \& Applications (IJOCTA), 6(2), pp. 75-83, (2016).

28. F. Evirgen, Conformable fractional gradient based dynamic system for constrained optimization problem, Acta Physica Polonica A, 132 (3), 1066-1069, (2017).

29. B. Batiha, M.S.M. Noorani, I. Hashim and E.S. Ismail, The multistage variational iteration method for a class of nonlinear system of ODEs, Phys. Scr., 76, pp. 388-392, (2007).

30. K. Schittkowski, More test examples for nonlinear programming codes, Springer, Berlin, (1987). 